https://doi.org/10.25312/2391-5137.14/2020_05rl

Radosław Lis (iD) https://orcid.org/0000-0002-6801-1799

Uniwersytet Technologiczno-Humanistyczny im. Kazimierza Pułaskiego w Radomiu e-mail: radoslaw.lis@gmail.com

\title{
Der politische Wortschatz - Merkmale der LTI
}

\section{Zusammenfassung}

Die vorliegende Studie untersucht den spezifischen Sprachgebrauch der Nationalsozialisten, erforscht die Strukturen und Inhalte der politischen Sprache und fragt nach ihren Absichten und Wirkungen. Die Quellengrundlage dieser Studie bezieht sich auf die von 1939 bis 1945 herausgegebene Besatzungspresse, die von den Deutschen für die Bevölkerung des Generalgouvernements gedruckt wurde.

Schlüsselwörter: LTI, Sprache, Generalgouvernement, Besatzungspresse

Sprache hatte im nationalsozialistischen Regime eine Vermittlungsfunktion, sie veranschaulichte und bewertete die außersprachliche Realität. Das ideologische Vokabular der Nationalsozialisten diente mit der Verwendung bestimmter Wendungen, Wörter und Begriffe als Instrument zur Analyse und Beurteilung ihrer politischen, wirtschaftlichen und gesellschaftlichen Umwelt. Darüber hinaus formulierten damitdie Nationalsozialisten ihre politischen Prioritäten und versuchten mittels der Sprache die eigene Sichtweise nicht nur im deutschen Volk, sondern auch in den unterworfenen Gebieten durchzusetzen. Deshalb war ihre politische Sprache somit ein Mittel gesellschaftlicher Kontrolle, da sie neben Inhalten auch gesellschaftliche Bewertungen vermittelte (Lis, 2009: 419).

Innerhalb der Taxonomie der politischen Sprache können zwei verschiedene Richtungen unterschieden werden: zum einen wird darunter die Anwendung der Sprache im Bereich der Politik zur Erreichung eines bestimmten Ziels verstanden sein, und zum anderen - der spezielle politische Wortschatz (Dieckmann, 1975: 47). Diese wiederum 
kann nach mehreren Kriterien Kriterien unterteilt werden. Eine solche Einteilung wurde vom Sprachwissenschaftler Gerhard Strauß vorgeschlagen, der den politischen Wortschatz in vier Lexemklassen einteilt:

1) Politische Fachtermini (Formula) der Institutions-, Verfahrens- und Organisationssprache, der Fachsprachen der verwalteten Sachgebiete, der Rechts- und Verwaltungssprache und andere,

2) Gesellschaftspolitische Termini der Ideologiesprache (Miranda, Credenda, Fahnen-, Stigmawörter),

3) Politische Metaphern, Euphemismen, okkasionelle Wortbildungen (Neologismen),

4) Termini der fächerübergreifenden Wissenschafts- und Bildungssprache (Strauß, 1986: 149).

Eine andere Unterteilung, die von Walther Dieckmann vorgeschlagen wurde, beinhaltet die Unterteilung der politischen Sprache in die folgenden Unterkategorien:

1) Ideologiesprache,

2) Institutionssprache,

3) Fachsprache der verwaltenden Sprachgebiete (Dieckmann, 1975: 47).

Joseph Klein, ein anderer Forscher, behauptet, dass Sprachmuster, die in der Politik häufig vorkommen, eine Kombination aus vier Hauptelementen sind:

1) dem Institutionsvokabular,

2) dem Ressortvokabular,

3) dem allgemeinen Interaktionsvokabular,

4) dem Ideologievokabular (Klein, 1989: 4).

Klein definiert das Institutionsvokabular als Bezeichnungen für die staatlichen Organisationen, politischen Institutionen, Parteinamen, staatliche und politische Rollen, kodifizierte Normierungen von politisch-institutionellen Handelns und auch für politische Handlungen, Prozesse und Zustände (Klein, 1989: 5).

Hinter dem Begriff Ressortvokabular verbergen sich vor allem Bezeichnungen aus den verschiedenen, ressortspezifischen Sachbereichen, wie z.B. auswärtige Beziehungen, Wirtschaft, Finanzen, Sozialpolitik, Umwelt, Justiz, Städtebau, Bildungswesen etc. (Klein, 1989: 6).

Und schließlich, in Bezug auf dasallgemeine Interaktionsvokabular, umfasst darunter die Definition unterschiedliche Ausdrücke zur Bezeichnung politischer Handlungen und Handlungsaspekte sowie sprachlicher Interaktionen (Klein, 1989: 7).

Das Ideologievokabular umfasst dagegen solche Wörter, mit deren Hilfe Politiker ihre Deutungen und Bewertungen der politisch-sozialen Welt, sowie ihre Prinzipien und Prioritäten formulieren. Dazu gehören vor allem Ausdrücke, die es dem Sprecher erlauben, was jeweils als grundlegende soziale Beziehungen und Formationen gilt zu artikulieren. Erwähnenswert sind auch Lexeme, in denen die von der Organisation favorisierten Prinzipien des politischen Lebens formuliert werden, wie auch solche lexikalischen Mittel, mit denen die grundlegenden Werte und Handlungsorientierungen zum Ausdruck kommen.

Ein unvermeidlich wichtiger Aspekt, der berücksichtigt werden muss, ist der Einfluss auf die Bürger durch bestimmte Sprachverwendungen im Bereich der Politik, denn in diesem Bereich das Wort ist ein mächtiges Instrument. Es kann im guten und schlechten Sinne, geschickt oder ungeschickt benutzt werden. Die Sprache der Politik ist zwar keines- 
wegs der entscheidende Aspekt der Politik, aber sie steht für eine wichtige Komponente im politischen Geschehen (Klaus, 1972: 9).

Die Manipulation und der spezifische Gebrauch der Sprache war jedoch keine Erfindung der Nationalsozialisten. Diese wurden im 16. Jahrhundert von Martin Luther geübt, denn er erkannte schon bald, sich in einer Welt abzufinden, in der die Machtausübung gerade auf Manipulation basiert (Lis, 2018: 274). Auch das nationalsozialistische Regime lernte rasch durch zahlreiche kleinere oder größere Manöver, das Verhalten seiner Staatsbürger zu seinen Gunsten zu ändern (Lay, 1999: 12).

Zweifellos ist eine eindeutige und umfassende Definition von Manipulation schwer zu bestimmen. Man könnte vielleicht, sehr allgemein definiert, sagen, Manipulation sei ein unerlaubtes Mittel zur Beeinflussung des Menschen (als Einzelwesen oder in der Gruppe) zum Zweck einer systematischen zielgerichteten Lenkung und Prägung des Bewusstseins, der Lebens- und Denkgewohnheiten, der Gefühlslagen (Lay, 1999: 20). Es sei aber darauf hingewiesen, Manipulation ist auch eine besondere Art des Sprachgebrauchs. Diese Besonderheit besteht darin, dass durch spezifische Sprachverwendung bestimmte Ziele angestrebt werden, die dem Partner ,,verborgen sind, verborgen bleiben sollen oder doch bleiben können“" (Mackensen, 1982: 208). Die Manipulation verwandelt die Sprache in ein Mittel für die einen, über die anderen zu herrschen. Unabhängig von der genauen Definition birgt sie jedenfalls die Möglichkeit, mit ihrer Hilfe Macht über andere zu gewinnen: das ist eine ihrer Grundfunktionen. Diese Art der politischen Einflussnahme auf die Menschen ist sicherlich notwendig, denn ohne sie wäre es nahezu unmöglich, politische Stabilität zu erreichen (Lay, 1999: 167).

Die Manipulation durch Sprache ist wohl der auffälligste und vermutlich auch der am häufigsten vorkommende Art und Weise des Versuchs, auf andere bewusst und berechnend bestimmenden Einfluss zu gewinnen. Es liegt nur an der List des Sprechers bzw. des Schreibers, ob sein Vorhaben ohne Weiteres erfolgreich ist. Meist übersehen die Hörer und Leser diese List (Mackensen, 1982: 96).

NachWahrheit und Sinn in den vom nationalsozialistischen Regime manipulierten politischen Aussagen oder Texten zu suchen, würde sich sicherlich als eine vergebliche Aufgabe erweisen, denn diese Elemente sind unsichtbar und unwirklich. Das ist das unvermeidliche Ergebnis, wenn man versucht, das Verhalten seiner Mitmenschen zu eigenem Nutzen zu ändern. Rücksichtlose Wortmanipulationen reduzierte die Sprache auf eine eitle Angelegenheit und auf ein bloßes Mittel und übte einen riesigen Einfluss auf das ganze damalige Schrifttum (Lay, 1999: 389).

Das nationalsozialistische Regime war sich dessen bewusst, dass Sprache viel mehr als nur ein Medium der Verständigung ist und dass die Rolle des pragmatischen Aspektes der Sprache im weltanschaulichen Kampf der Nationalsozialisten nicht unterschätzt werden darf. Hier handelte es sich um eine besondere Form der Sprachregelung, die letzten Endes der Irreführung diente. Mittels einer zum Teil bis ins Detail geregelten Wortwahl über die gleichgeschalteten Medien wurde versucht, eine neue nationalsozialistische Wirklichkeit zu schaffen, die wahren Absichten und die tatsächlichen Ziele und Handlungen des NS-Regimes zu verschleiern, gleichzeitig auch die Menschen in den Dienst eines Systems zu stellen, welches ihren eigenen Interessen zuwiderhandelte (Klaus, 1972: 40). 
Im Dritten Reich vollzog sich eine Durchdringung des gesamten öffentlichen und privaten Lebens und Schrifttums. Unter dem Einfluss des Nationalsozialismus verwirklichte sich eine außerordentlich starke und weitgehende Veränderung der deutschen Sprache, ungeachtet dessen, ob es die Form von Propagandareden, Zeitungsartikeln aller Art, wissenschaftlichen Büchern oder privaten Korrespondenzen nahm (Seidel, 1961: 2).

Eines der wesentlichen, hervorzuhebenden Merkmale für den nationalsozialistischen Sprachgebrauch ist seine allgegenwärtige Rhetorizität, die aber nicht im Sinne eines klassischen juristischen oder philosophischen Redners vorkommt, sondern Volksrednern und Demagogen ähnelt. „Hitler spürte in der deutschen Sprache eine andere Musik als die von Goethe, Heine oder Mann auf. Und das deutsche Volk gab dem Gebrüll des Mannes einen massiven Widerhall“" (Steiner, 2014: 129).

Es werden nie verschiedene Positionen oder Rechte abwägend eingebracht, nie nach Objektivität und Wahrheit gestrebt. Das einzige Ziel war, die Massen zu gewinnen, wofür jedes Mittel recht ist. Appelliert wird an den Instinkt der Angesprochenen, und nicht an ihren Verstand, denn rational ist die Argumentation in keinem Moment (Hundhausen, 1975: 187).

Der Kern des Problems liegt hier also nicht in einem gepflegten, auf Wissen und Verstand basierenden Stil mit ausgewogenem Aufbau, These und Antithese, sondern in einer Art Vergewaltigung des Sprachgeistes in Deutschland, wobei ganz offensichtlich, zumeist mit Unterstützung der Presse, das Bewusstsein zahlreicher Bürger manipuliert wird, um sie an einen ganz bestimmten Punkt zu bringen. Das Verhalten des Volkes soll so umgestimmt werden, dass es einerseits die Machttaten des nationalsozialistischen Regimes akzeptiert oder sogar ausdrücklich fordert und andererseits im gemeinsamen Hass, in der gemeinsamen Angst zu so etwas findet wie „einem Zusammengehörigkeitsgefühl, das dann ein politisches Gebilde auf sich beziehen kann“ (Lay, 1999: 179).

Sicherlich muss man zu dem Schluss kommen, dass alles, was sich zwischen Menschen abspielt, von Sprache geführt oder verführt wird. Auch die Geschichte wird von durch Worte konstruiert, mehr denn von Männern und Taten. Die Sprache darf somit nicht als statisch wahrgenommen werden, denn sie muss einer sich ständig wandelnden Lebenswirklichkeit Rechnung tragen (Lay, 1999: 389). Die Sprache bot den Nationalsozialisten einen Spielraum, den sie für die Durchsetzung ihrer Denkweise und Ideologie sowie für die Realisierung ihrer politischen Pläne nutzen konnten. Und dieser Eingriff des nationalsozialistischen Staates in die lebendige Volkssprache teilten die typischen Eigenschaften von den Charakter eines systematischen Vorgehens (Klaus, 1972: 131).

Selbstverständlich hatten die Nationalsozialisten ihr bestimmtes Vokabular, gereift mit zahlreichen Sprachschöpfungen und Sprachverdrehungen, und eine bestimmte öffentliche Rhetorik, die ihr Programm, zusammen mit der Gewalt und dem propagandistisch ausgerichteten Stil, untermauerten. Es kann zweifelsfrei festgestellt werden, dasses gewisse Eigenschaften gibt, die für alle totalitären Sprachen typisch sind. Eine genaue Analyse der Sprache des Nationalsozialismus lässt eine Reihe von solchen Merkmalen erkennen, die als charakteristisch für diese Sprache gelten können, denn der Sprachgebrauch im Dritten Reich weist mit seinen Umdeutungen und Umwertungen einige Auffälligkeiten auf, was insbesondere dem NS-Wortschatz seinen spezifischen Klang gibt (Kołtunowski, 1981: 199). 
Insgesamt bestand die nationalsozialistische Vorgehensweise darin, vorhandene Ideen und Begriffe zu übernehmen, sie an gängige weltanschauliche Vorstellungen anzuknüpfen, alte ideologisch besetzte Wörter aufzugreifen, die Wortbedeutung aber zu ändern (Straßner, 2012: 175).

„Das Gift ist überall. Im Trinkwasser der LTI wird es verschleppt, niemand bleibt davon verschont" (Klemperer, 1949: 102). Die Reichweite der offiziellen Sprache, die zahlreiche Begriffe und Gefühle schändete und vergiftete, hatte damit weit mehr Einfluss und reichte über ihre Verwendung in Reden der Parteioberen und Funktionären in Verordnungen, Gesetzen, Presseanweisungen, Rundfunksendungen und Zeitungen hinaus. Die von den Nationalsozialisten mit mehr oder weniger Zwang durchgesetzten Sprachformen wurden zu einem Bestandteil der Allgemeinsprache, und infolgedessen auch der Denkstrukturen weiterer Teile der deutschen Bevölkerung. Die Einzelwörter, Redewendungen, Satzformen undie millionenfachen Wiederholungen wurden automatisch und unbewusst inkorporiert (Schmitz-Berning, 2010: 7).

$\mathrm{Zu}$ den auffälligsten sprachlich-stilistischen Merkmalen der Lingua Tertii Imperii gehörten u.a.:

1) Ausdrücke aus wissenschaftlicher Fachsprache (vor allem aus den Bereichen Militär, Naturwissenschaft, Technik und Religion), die in andere Lebensbereiche übertragen wurden und somit eine andere Bedeutung erhielten,

2) Euphemismen (Hüllwörter), deren Hauptaufgabe war, grausame Taten der Nationalsozialisten zu verdecken oder zu verharmlosen,

3) Superlative zur Bezeichnung einzelner Personen oder Personengruppen und deren Leistungen,

4) Verstärktes, falsches Pathos in der Ausdrucksform, das zur Betonung des Gefühls beitragen sollte,

5) Fremdwörter,

6) Neologismen (Lis, 2008: 23).

Die oben genannten Beispiele für die Vergiftung der deutschen Sprache sollen hier lediglich als eine eingeschränkte Auswahl dessen betrachtet werden, womit das NS-Regime den öffentlichen Diskurs im Dritten Reich überschwemmt hat.

Natürlich ist die Sprache an sich selbst nicht schändlich. Als Machwerk des Menschen kann sie aber besonders gefährlich sein. „Worte können sein wie winzige Arsendosen“, denn sie ,werden unbemerkt verschluckt, sie scheinen keine Wirkung zu tun, und nach einiger Zeit ist die Giftwirkung doch da" schrieb Victor Klemperer 1949 in seinem berühmten Werk LTI über die Sprache im Nationalsozialismus. Diese kann das menschliche Gefühl lenken, das seelische Wesen steuern, je unbewusster man sich ihr überlässt (Klemperer, 1949: 21). Es kommt also darauf an, dass je gedankenloser die Sprache benutzt wird, desto mehr kann Macht sie auf das Denken und Fühlen ausüben. Sie kann, wenn sie erst einmal vergiftet und manipuliert ist, ihr Gift entfalten. Und die nationalsozialistischen Machthaber wählten und benutzten die Sprache bewusst als ein Mittel für die Manipulation der Massen (Kołtunowski, 1980: 155). 


\section{Literatur}

Dieckmann W. (1975), Sprache in der Politik. Einführung in die Pragmatik und Semantik der politischen Sprache, Heidelberg.

Hundhausen C. (1975), Propaganda. Grundlagen-Prinzipien-Materialien-Quellen, Berlin. Klaus G. (1972), Sprache der Politik, Berlin.

Klein J. (1989), Wortschatz, Wortkampf, Wortfelder in der Politik, [in:] Klein J. (Red.): Politische Semantik. Beiträge zur politischen Sprachverwendung, Opladen 1989.

Klemperer V. (1949), LTI. Notizbuch eines Philologen, Berlin.

Kołtunowski P. (1980), Niemcy, Żydzi i Polacy w Krakauer Zeitung. Przyczynek do badań nad językiem narodowego socjalizmu w Niemczech hitlerowskich, „Lubelskie Materiały Neofilologiczne", Nr 9, SS. 155-164.

Kołtunowski P. (1981), Psychologiczna rola języka narodowego socjalizmu w propagandzie hitlerowskiej na podstawie Krakauer Zeitung, „Lubelskie Materiały Neofilologiczne”, Nr 10, SS. 199-211.

Lay R. (1999), Manipulation durch die Sprache, Berlin.

Lis R. (2008), Propagandasprache im Dritten Reich anhand von der NS-Besatzungspresse im Generalgouvernement, München.

Lis R. (2009), Sprache als politisches Instrument, „Studia Niemcoznawcze”, Nr XLII, SS. 417-420.

Lis R. (2018), Od Lutra do Goebbelsa - rola mediów w propagandzie na przestrzeni wieków, „Studia Niemcoznawcze”, nr LXI, SS. 271-278.

Mackensen L. (1982), Verführung durch Sprache, München.

Schmitz-Berning C. (2010), Vokabular des Nationalsozialismus, Berlin.

Seidel E. / Seidel-Slotty, I. (1961), Sprachwandel im Dritten Reich. Eine kritische Untersuchung faschistischer Einflüsse, Halle.

Steiner G. (2014), Das hohle Wunder, [in:] Steiner G. (Red.), Sprache und Schweigen: Essays über Sprache, Literatur und das Unmenschliche, Frankfurt am Main.

Straßner E. (2012), Ideologie - Sprache - Politik, Tübingen.

Strauß G. (1986), Schwere Wörter in der Politik, [in:] Strauß G. (Red.), Der politische Wortschatz. Zur Kommunikations- und Textsortenspezifik, Tübingen.

\section{Abstract \\ The political language - characteristics of the LTI}

The following article presents the characteristics of the language of the Third Reich, taking into account the structure and content of the language of politics. The paper also analyzes the plans of national socialists in this regard and the impact of the language constructed on its recipients. The source material for this analysis is the occupation press published from 1939 to 1945, printed by the Germans for the residents of the General Government.

Keywords: LTI, language, General Government, occupation press 


\section{Streszczenie}

\section{Język polityki - charakterystyka języka Trzeciej Rzeszy}

Tematem niniejszego artykułu jest próba analizy języka propagandy hitlerowskiej. Bazą źródłową, wykorzystaną pod kątem zasygnalizowanej problematyki, są wybrane egzemplarze wydawanego w Generalnym Gubernatorstwie dziennika „Krakauer Zeitung”, wplecionego w latach 1939-1945 w system propagandy hitlerowskiej i będącego jednym z jej najistotniejszych ogniw. Dziennik ten miał być organem kierownictwa politycznego oraz nosicielem woli politycznej i misji kulturowej narodu niemieckiego.

Słowa kluczowe: język Trzeciej Rzeszy, Generalne Gubernatorstwo, prasa okupacyjna 